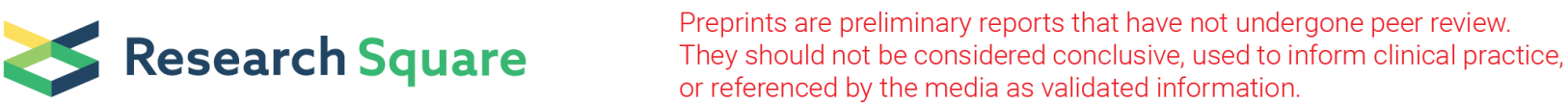

\section{Hepatitis $B$ virus infection among men who have sex with men and transgender women living with or at risk for HIV: A cross sectional study in Abuja and Lagos, Nigeria.}

Olusegun A. ADEYEMI

University of Maryland Baltimore

Andrew MITCHELL

University of Maryland Baltimore

Ashley SHUTT

University of Maryland Baltimore

Trevor A. CROWELL

US Military HIV Research Program

Nicaise NDEMBI

Institute of Human Virology Nigeria

Afoke KOKOGHO

US Army Medical Research Directorate- Africa

Habib O. RAMADHANI

University of Maryland Baltimore

Merlin L. ROBB

Henry M Jackson Foundation for the Advancement of Military Medicine Inc

Stefan D. BARAL

Johns Hopkins University Bloomberg School of Public Health

Julie A. AKE

US Military HIV Research Program

Manhattan E. CHARURAT

University of Maryland Baltimore

Sheila PEEL

US Military HIV Research Program

Rebecca G Nowak ( $\sim$ mowak@ihv.umaryland.edu )

University of Maryland Baltimore https://orcid.org/0000-0001-8404-4569

Research article 
Keywords: HBV DNA, Sexual and Gender Minorities, Antiretroviral Therapy, sub-Saharan Africa

Posted Date: March 3rd, 2020

DOI: https://doi.org/10.21203/rs.3.rs-15740/v1

License: (c) (1) This work is licensed under a Creative Commons Attribution 4.0 International License. Read Full License

Version of Record: A version of this preprint was published at BMC Infectious Diseases on July 6th, 2021. See the published version at https://doi.org/10.1186/s12879-021-06368-1. 


\section{Abstract}

Background Despite the development of a safe and efficacious hepatitis B vaccine in 1982, the hepatitis $B$ virus (HBV) remains a public health burden in sub-Saharan Africa. Due to shared risk factors for virus acquisition, men who have sex with men (MSM) and transgender women (TGW) living with HIV are at increased risk of HBV. We estimated the prevalence of HBV and associated factors for MSM and TGW living with or without HIV in Nigeria.

Methods Since March 2013, TRUST/RV368 has recruited MSM and TGW in Abuja and Lagos, Nigeria using respondent driven sampling. Participants with HIV diagnosis, enrollment as of June 2015, and available plasma were retrospectively tested for hepatitis B surface antigen and HBV DNA. Logistic regression models were used to estimate odds ratios (ORs) and 95\% confidence intervals (Cls) for factors associated with prevalent HBV infection.

Results A total of 717 MSM and TGW had a median age of 25 years (interquartile range [IQR]: 21-27), 5\% self-reported HBV vaccination, $61 \%$ were living with HIV, $10 \%$ had prevalent $\mathrm{HBV}$ infection and $6 \%$ were HIV-HBV co-infected. HIV mono-infected as compared to HIV-HBV co-infected had a higher median CD4 T cell count [425 (IQR: 284-541) vs. 345 (IQR: 164-363) cells/mm $3, p=0.03$ ] and a lower median HIV RNA viral load [4.2 (IQR: 2.3-4.9) vs. 4.7 (IQR: 3.9-5.4) log $10 \mathrm{copies} / \mathrm{mL}, \mathrm{p}<0.01]$. The only factor independently associated with HBV was self-report of condomless sex at last anal intercourse (OR: 2.2, 95\% Cl: 1.3, 3.6). HIV infection was not independently associated with HBV (OR: 1.0, 95\% Cl: 0.7-1.6).

Conclusion HBV prevalence was moderately high but did not differ by HIV in this cohort of MSM and TGW. Condomless sex was associated with elevated HBV risk, reinforcing the need to increase communication and education on condom use among key populations in Nigeria. Evaluating use of concurrent HIV antiretroviral therapy with anti-HBV activity may confirm the attenuated HBV prevalence for those living with HIV.

\section{Background}

Despite the development of a safe and efficacious hepatitis B vaccine in 1982 [1], the hepatitis B virus (HBV) remains a public health burden in sub-Saharan Africa with a reported prevalence of about $6 \%[2,3]$. While mortality in sub-Saharan Africa from the World Health Organization (WHO)-targeted infectious diseases (i.e. HIV, tuberculosis and malaria) is now declining, morbidity and mortality due to viral hepatitis is increasing [2]. Globally, HBV is the leading cause of end-stage liver disease (ESLD) and hepatocellular carcinoma (HCC) [4]. In 2015, HBV infections were responsible for 887,000 deaths, in addition to 257 million individuals living with chronic HBV infections worldwide [5].

In resource-limited settings, enzyme immunoassays detecting HBV surface antigen (HBsAg) are commonly used as a standard of care diagnostic test for active HBV infection. However, concerns have been raised about the use of this test for those co-infected with HBV and HIV, in whom the targeted HBV immune markers might be suppressed [6, 7]. A more sensitive diagnostic assay that quantifies HBV-DNA 
rather than circulating antibodies is the polymerase chain reaction (PCR)-based molecular diagnostic assay that has served as a confirmatory and treatment progress-monitoring test for active HBV $[8,9]$.

Due to shared risk factors for acquisition, HBV is common in persons living with HIV (PLWHs) [10]. Furthermore, men who have sex with men (MSM) are 2 to 4 times [2] and 3 to 6 times [11] more likely to be infected with HBV and HIV, respectively, as compared to reproductive aged adults [12]. HBV-HIV coinfection is associated with a 14 fold increased risk in progressing to ESLD compared to mono-infection with either virus [13] and is also associated with increased risk of hospitalization for non-liver-related causes $[14,15]$. As the advent of ubiquitous combination antiretroviral therapy has improved life expectancies of MSM and transgender women (TGW) living with HIV, these individuals are now at higher risk of developing chronic complications from comorbid infections such as HBV $[16,17]$.

In Nigeria, prevalence studies of HBV range between 7-14\% among reproductive aged adults [18, 19], and in Calabar, Southeast Nigeria, $69 \%$ of patients with ESLD tested positive for HBsAg [20]. HBV prevalence among PLWH range from 4-8\% [21-23] and as high as 10 and18\% for MSM in Ibadan and Lagos, Nigeria, respectively [24]. The objective of these analyses was to investigate the prevalence and factors associated with PCR-diagnosed HBV among MSM and TGW living with or at risk for HIV in Nigeria. We hypothesized that HIV infections would be associated with increased odds of HBV infection in Nigerian MSM and TGW.

\section{Methods}

\section{Study design and population}

The TRUST/RV368 cohort study used respondent-driven sampling (RDS) to recruit MSM and TGW between March 2013 and February 2020 in Abuja and between April 2014 and May 2018 in Lagos as previously described [25]. Eligibility criteria included the following: (1) assigned male sex at birth, (2) history of insertive or receptive anal intercourse with another man in the past 12 months, (3) age $\geq$ 16 years in Abuja and $\geq 18$ years in Lagos, (4) possession of a valid RDS coupon, and (5) ability and willingness to provide written informed consent. In addition to these criteria, participants included in these cross-sectional analyses had an enrollment visit at the TRUST/RV368 clinics before June 2015, an HIV diagnostic result, and plasma for retrospective HBV screening. During enrollment evaluations, spaced approximately two weeks apart, consented participants underwent HIV counseling and testing and provided demographic, behavioral and clinical data through in-person interviews with trained staff using a standardized questionnaire. In addition, participants received physical examinations and sexually transmitted infection (STI) diagnostics [26].

\section{Laboratory procedures}

HBsAg screening was completed using the Genescreen HBsAg 3.0 EIA assay (Bio-Rad Laboratories, Redmond, WA). To confirm the presence of HBV and to quantify DNA, we performed in vitro PCR using 
the Abbott RealTime HBV assay (Abbott Molecular, Des Plaines, IL). Participants who tested positive for HBsAg with detectable HBV DNA were considered HBV-infected.

Whole blood was tested for HIV using a parallel series of rapid tests (Alere Determine, Waltham, MA and Trinity biotech Uni-Gold HIV, Wicklow, Ireland). In cases of discordance, a 3rd rapid test (Chembio Diagnostics HIV-1/2 Stat Pak test, Medford, NY) was used to confirm or exclude HIV infection as outlined by the parallel testing algorithm for at-risk individuals in Nigeria [27]. For participants living with HIV, HIV RNA was quantified using the Ampliprep/COBAS TaqMan HIV-1 Test (Roche Molecular Diagnostics, Pleasanton, CA) and CD4 counts using the Partec CyFlow Counter (Sysmex, Kobe, Japan). Voided urine and rectal swabs were tested for Neisseria gonorrhoeae (NG) and Chlamydia trachomatis (CT) using the Aptima Combo 2 CT/ NG Assay (Hologic, San Diego, CA). All participants testing positive for HIV or other STIs were offered appropriate treatment regardless of CD4 count.

\section{Statistical analyses}

The primary outcome of this investigation was prevalent HBV infection, and the main explanatory variable was HIV infection status, categorized as infected or uninfected. Potential cofactors evaluated included age (16-20, 20-25, 25-60 years), site (Abuja, Lagos), education (primary, secondary, tertiary), marital status (ever, never, other), willingness to access health care services (yes,no), HBV vaccination status (yes, no), sexual orientation (homosexual or bisexual), sexual positioning (insertive only or any receptive), condom use during last anal sex (yes, no), concurrent sexual partners (yes, no), prevalent STIs (any urethral or rectal NG or CT), and vaginal sex in the last year (yes, no).

Distributions of demographic characteristics were compared by HBV status using Pearson's Chi-square and Fisher's exact tests for categorical variables. Wilcoxon rank sum test was used to compare continuous variables. Bivariate and multivariable logistic regression models were used to estimate odds ratios (ORs) and 95\% confidence intervals (Cls) for the association between HIV and other factors with HBV. Confounders were identified based on biological plausibility, statistical associations with exposure and outcome $(p<0.10)$, and at least a $10 \%$ change in the crude beta estimates of the main independent variable. Potential confounders were entered into the multivariable model via forward stepwise selection and retained based on signficance after likelihood ratio testing [28]. Missing observations accounted for less than $4 \%$ of the total sample and were retained in the models with indicators. A p-value less than 0.05 was considered statistically significant and a p-value less than 0.10 was suggestive of a trend. Characteristics of individuals who were included in the sample versus excluded because of incomplete data were compared using Pearson's Chi-square test. Data analyses were performed using SAS version 9.4 (SAS Institute, Cary, NC USA)

\section{Results}

Of the 771 participants enrolled from March 2013 to June 2015, 717 had real-time HIV and retrospective HBV testing results available and were included in our analyses. Out of these, $438(61 \%)$ were HIVinfected and 69 (10\%) were HBV-infected, including 41 (6\%) with HIV-HBV co-infection. HIV-HBV coinfected participants had a lower median CD 4 T cell counts of 425 (interquartile range [IQR]: 284-541) 
cells $/ \mathrm{mm}^{3}$ than HIV mono-infected participants, 345 (IQR: $164-363$ ) cells $/ \mathrm{mm}^{3}(\mathrm{p}=0.03)$. Similarly, HIVHBV co-infected participants had a higher median HIV RNA viral load of 4.7 (IQR: 3.9-5.4) $\log _{10}$ copies/mL than HIV mono-infected participants, 4.2 (IQR: 2.3-4.9) $\log _{10}$ copies/mL $(p<0.01)$. Among participants living with HIV, 51\% (223/438) had been told by a physician or healthcare provider before enrolling in TRUST/RV368 that they needed to begin treatment for HIV. Of these participants, $24 \%$ (107/438) self-reported receiving medication to treat their HIV from a healthcare facility or pharmacy, but not all were able to recall the specific medication regimen they received prior to enrollment.

The median age of participants included in these analyses was 25 years with an IQR of 22 to 27 years. Sixty-two percent of participants identified as bisexual and $75 \%$ engaged in any receptive sex (Table 1 ). Eighty-seven percent had never married, and $93 \%$ had at least a secondary school (high school equivalent) education. Regarding health services, $63 \%$ were willing to access care but only $5 \%$ selfreported HBV vaccination. Compared to HIV-uninfected persons, HIV-infected persons were more likely to be older, married, and engaged in any receptive sex. However, HIV-infected MSM and TGW were less willing to access health care services. The prevalence of HBV in the study population did not differ between those living with or without $\mathrm{HIV}(9 \%[95 \% \mathrm{Cl} 6.6,12.1]$ vs. $10 \%[95 \% \mathrm{Cl} 6.5,13.6]$, respectively, $\mathrm{p}=$ 0.8 ). After adjusting for potential confounders, HIV was not associated with HBV prevalence (adjusted OR: 1.0, 95\% Cl: 0.7, 1.6) (Table 2).

Those who had not used a condom during last anal sex were twice as likely to be infected with HBV (OR: $2.2,95 \% \mathrm{Cl}: 1.3,3.6$ ) as compared to those who had used a condom in the unadjusted analysis (Table 2). In the multivariable analysis, condomless sex remained an independent factor and was associated with a two-fold increased odds of HBV infection as compared to those who used condoms during last anal sex (adjusted OR: 2.2, 95\% Cl: 1.3, 3.6). Although not significant, self-reported homosexual orientation independently trended towards an association with HBV infection (adjusted OR: 1.5, 95\% Cl: 0.9-2.6). Age, marital status, willingness to access healthcare services, HBV vaccination, sexual position, concurrent sexual partners, prevalent bacterial STIs, and vaginal sex in the last year were not associated with HBV. After comparing those included and excluded from the analyses, the retained individuals were less willing to access health care services and more likely to self-identify as homosexual as compared to bisexual (all $p<0.05)$.

\section{Discussion}

The HBV prevalence of $10 \%$ observed among MSM and TGW in our study was comparable to those reported by cross-sectional studies of MSM in Lagos, Nigeria (10\%) [24] and Brazil (11\%) [29] but higher than Tanzania (5\%) [30]. Our estimates were slightly higher than previous estimates of PLWH (4-8\%) in Nigeria with unknown sexual orientation [21-23]. However, our results were lower than those from a study conducted in Ibadan (Southwest Nigeria) where the reported HBV prevalence among MSM was $18 \%$ [24]. The higher estimates from the Ibadan study [24] may be attributed to an older age, smaller sample size, and overestimation from enzyme-linked immunosorbent assay based methods commonly used in resource-limited settings [6]. 
In contrast to our hypothesis, the prevalence of HBV was not higher among MSM and TGW living with HIV. This finding is consistent with a prior systematic review that reported an $8 \%$ pooled prevalence of HBsAg that did not differ by HIV among a population of Malawian adults (OR:1.2, 95\% Cl: $0.8,1.6)[31]$. A small proportion of our participants self-reported taking doctor or pharmacy prescribed medicine for their HIV infection before study entry. If our participants were prescribed antiretroviral therapy (ART) with antiHBV activity, this may have reduced HBV infectivity [32,33]. Multiple studies have shown that tenofovir disoproxil fumarate (TDF) containing ART regimen, a first-line regiment in Nigeria[34], have significant anti-HBV activity [35-37]. A systematic review and network meta-analysis of randomized controlled trials [36] confirmed that TDF and tenofovir alafenamide fumarate (TAF) are the most effective ART regimens for HBV virologic suppression. In the Multicenter AIDS Cohort Study, MSM receiving effective ART (HIV RNA level < 400 copies $/ \mathrm{mL}$ ) had the same risk for incident HBV infection as MSM not living with HIV [32]. This pre-exposure prophylaxis effect of TDF containing regimens for HBV co-infections was further confirmed in the Swiss HIV Cohort where there was a reduction of HBV incidence among those on effective ART for their HIV infections [33]. TDF containing regimens may have virally suppressed HBV and prevented transmission for those living with HIV in our cohort.

Although our study did not find a significant association between HIV and HBV prevalence, it offers some insight into how the use of condoms and sexual activities may affect HBV infection. In our

TRUST/RV368 cohort, condom usage has been suboptimal and this is a population known to have a high burden of human immunodeficiency virus [38], anorectal STIs [26], and sexual risk practices [39]. Consistent with our findings, the Swiss HIV Cohort study reported condomless sex increased the hazard of HBV acquisition 2-fold among those living with HIV independent of TDF-containing regimens [33]. In Nigeria, Adebajo et al. found an increased odds of HBV infection in Nigeria among MSM engaging in condomless sex that was predominantly for the insertive as opposed to receptive sexual position [24]. In our study, sexual positioning was not associated with HBV infection. Also in the study by Adebajo et al., participants who identified as bisexual were significantly more likely to have HBV (OR:5.2, $95 \mathrm{Cl}: 3.04,9.0)$ [24], whereas our findings suggested the opposite, demonstrating a 2-fold increased prevalence of HBV for those who self-identified as homosexual. Socio-cultural differences in reporting of sexual practices between the North-central (Abuja) and Southwestern regions (Ibadan and Lagos) of Nigeria may affect how participants were categorized [40]. Despite sexual position or sexual orientation, culturally acceptable methods to communicate the benefits of condoms during anal sex for unvaccinated individuals could reduce the risk of HBV and associated morbidities such as ESLD and HCC.

Self-reported vaccination coverage among MSM and TGW was $5 \%$ and insufficient in preventing active HBV infection [5]. Routine childhood HBV vaccination in Nigeria was only enacted 15 years ago as part of the expanded immunization program and possibly explains the low vaccination coverage [41]. Most highincome countries with effective vaccination programs have much lower estimates of active HBV. A nationwide, multi-center prospective German study of HIV-infected MSM reported active HBV prevalence as $2 \%$, cleared HBV as $27 \%$ and occult HBV as $8 \%$ [42]. Similarly, a Danish studyfrom a large tertiary hospital evaluating HBV infection and vaccine coverage among MSM reported active HBV infection as $1 \%$, cleared infection as $7 \%$, and HBV vaccinated as $14 \%$ [43]. These studies highlight the reduction in 
active HBV infection that is possible if a catch-up vaccination strategy were implemented for at-risk individuals in low and middle-income countries.

Our study had some limitations. With relatively small prevalence estimates, the multivariable model was not sufficiently powered to detect independent associations between HBV infection and known demographic and behavioral factors. However, most associations between sexual behaviors such as homosexual orientation and exclusively male partners with HBV infection aligned with the direction of the study hypothesis. Although, because more MSM who self-identified as homosexual were retained in our analyses, our prevalence estimates may have been an overestimate because of enrichment of those engaging in high risk sexual practices [38]. HBV vaccination was self-reported and it was unclear who had been exposed to HBV from vaccination versus a prior infection, as testing for HBV surface antibody was unavailable due to limited sample volume. Due to uncertainty regarding participants' past exposure to specific ART regimens, we were unable to assess whether HBV prevalence was lower among those living with HIV and exposed to TDF-containing regimens. The temporality of associations between HIV and HBV could not be determined because of the cross-sectional study design. Lastly, MSM and TGW originated from two cities in Nigeria and results from this study may not be generalizable to other regions of Nigeria.

\section{Conclusions}

HBV prevalence was moderately high and comparable to other studies of MSM but did not differ by HIV status. Condomless anal intercourse elevated the risk of HBV, reinforcing the need to increase communication and education on HBV and its potential long-term complications. Further studies are needed to confirm whether the attenuated HBV prevalence among MSM and TGW living with HIV was the result of TDF-containing ART regimens with anti-HBV activity.

\section{Abbreviations}

HBV: Hepatitis B Virus; MSM: Men who have sex with men; TGW: Transgender women; OR: Odds ratio; Cls: Confidence intervals; WHO: World Health Organization; ELSD: End stage liver diseases; HCC: Hepatocellular carcinoma; HBsAG: Hepatitis B surface antigen; PCR: Polymerase chain reaction; PLWHs: Persons living with HIV; RDS: Respondent driven sampling; STI: Sexually transmitted infections; NG: Neisseria gonorrhoeae; CT: Chlamydia trachomatis; IQR: Interquartile range; ART: antiretroviral therapy ; TDF: Tenofovir Disoproxil Fumarate; TAF: Tenofovir Alafenamide Fumarate; DNA; deoxyribonucleic acid; RNA: Ribonucleic acid

\section{Declarations}

\section{Ethics approval and consent to participate}


The study was approved by the University of Maryland Baltimore Institutional Review Board (IRB), Federal Capital Territory Health Research Ethics Committee, Nigerian Ministry of Defence Health Research Ethics Committee, and Walter Reed Army Institute of Research IRB. The investigators have adhered to the policies for protection of human subjects as prescribed in AR-70. All respondents gave both written and verbal consent to participate in this study.

\section{Consent for publication}

Not Applicable

\section{Availability of data and materials}

In order to protect the clinic staff and the participants from stigma and criminalization of same sex behavior, the data has not been made publicly available. The data is available from the corresponding author on request.

\section{Competing interests}

Dr. Stefan D. Baral is a section editor as part of the BMC Infectious Diseases editorial board. All other authors declare no competing interests.

\section{Funding}

The research reported in this publication was supported by funding from the U.S National Institutes of Health [R01 MH099001, R01 Al1209143, R01 MH110358, K07CS225403]; a cooperative agreement between the Henry M. Jackson Foundation for the Advancement of Military Medicine, Inc., and the U.S. Department of Defense [W81XWH-11-2-0174, W81XWH-18-2-0040]; Fogarty Epidemiology Research Training for Public Health Impact in Nigeria program [D43TW010051]; and the President's Emergency Plan for AIDS Relief through a cooperative agreement between the Department of Health and Human Services/Centers for Disease Control and Prevention, Global AIDS Program, and the Institute for Human Virology-Nigeria [NU2GGH002099]. The views expressed are those of the authors and should not be construed to represent the positions of the U.S. Army or the Department of Defense or the Department of Health and Human Services. The funding bodies had no role in the study design, collection, analysis, interpretation of data or in writing the manuscript.

\section{Authors' contributions}

OAA, TAC, NN, SDB, MEC, SP, RGN made substantial contributions to the conception, design, drafting and revising of the manuscript. AM, AS, AK, HOR, MLR, JAK made substantial contributions to data acquisition, analysis and interpretation. All authors read and approved the final manuscript. All authors are accountable for the accuracy and integrity of this work.

Acknowledgment: We thank the study participants and staff for their commitment and contributions to TRUST/RV386 study. The TRUST/RV368 Study Group includes Principal Investigators: Manhattan 
Charurat (IHV, University of Maryland, Baltimore, MD, USA), Julie Ake (MHRP, Walter Reed Army Institute of Research, Silver Spring, MD, USA); Co-Investigators: Aka Abayomi, Sylvia Adebajo, Stefan Baral, Trevor Crowell, George Eluwa, Charlotte Gaydos, Afoke Kokogho, Jennifer Malia, Olumide Makanjuela, Nelson Michael, Nicaise Ndembi, Rebecca Nowak, Oluwasolape Olawore, Zahra Parker, Sheila Peel, Habib Ramadhani, Merlin Robb, Cristina Rodriguez-Hart, Eric Sanders-Buell, Sodsai Tovanabutra, Sandhya Vasan; Institutions: Institute of Human Virology at the University of Maryland School of Medicine (IHVUMB), Johns Hopkins Bloomberg School of Public Health (JHSPH), Johns Hopkins University School of Medicine (JHUSOM), U.S. Military HIV Research Program (MHRP), Walter Reed Army Institute of Research (WRAIR), Henry M. Jackson Foundation for the Advancement of Military Medicine (HJF), Henry M. Jackson Foundation Medical Research International (HJFMRI), Institute of Human Virology Nigeria (IHVN), International Centre for Advocacy for the Right to Health (ICARH), The Initiative for Equal Rights (TIERS), Population Council (Pop Council) Nigeria.

\section{References}

1. MacKellar DA, Valleroy LA, Secura GM, McFarland W, Shehan D, Ford W, et al. Two decades after vaccine license: Hepatitis $B$ immunization and infection among young men who have sex with men. Am J Public Health. 2001;91:965-71.

2. World Health Organization. Global hepatitis report, 2017. 2017.

3. Platt L, French CE, Mcgowan CR, Sabin K, Gower E, Trickey A, et al. Prevalence and burden of HBV coinfection among people living with HIV: A global systematic review and meta-analysis. J Viral Hepat. 2019; September:1-22.

4. Lavanchy D. Hepatitis B virus epidemiology, disease burden, treatment, and current and emerging prevention and control measures. J Viral Hepat. 2004;11:97-107.

5. World Health Organization. Media centre New hepatitis data highlight need for urgent global response. 2018;:2-5.

6. Yetunde A, Bakarey AS. Evaluation of performance testing of different rapid diagnostic kits in comparison with EIAs to validate detection of hepatitis $B$ virus among high risk group in. $J$ Immunoass Immunochem. 2018;39:218-27.

7. Amini A, Varsaneux O, Kelly H, Tang W, Chen W, Boeras DI, et al. Diagnostic accuracy of tests to detect hepatitis $B$ surface antigen: A systematic review of the literature and meta-analysis. BMC Infect Dis. 2017;17 Suppl 1.

8. Zou S, Stramer SL, Dodd RY. Donor Testing and Risk: Current Prevalence, Incidence, and Residual Risk of Transfusion-Transmissible Agents in US Allogeneic Donations. Transfus Med Rev. 2012;26:119-28.

9. Ghosh S, Sow A, Ndow CGAJG, Njie R, Diop STM, Kane SMCT, et al. Implementation of an in- house quantitative real- time polymerase chain reaction method for Hepatitis B virus quantification in West 
African countries. J Viral Hepat. 2016; March:897-904.

10. Chun HM, Roediger MP, Hullsiek KH, Thio CL, Agan BK, Bradley WP, et al. Hepatitis B virus coinfection negatively impacts HIV outcomes in HIV seroconverters. J Infect Dis. 2012;205:185-93.

11. Beyrer C, Baral SD, Van Griensven F, Goodreau SM, Chariyalertsak S, Wirtz AL, et al. Global epidemiology of HIV infection in men who have sex with men. Lancet. 2012;380:367-77.

12. Richard Parker, Shivananda Khan PA. Conspicuous by their absence? Men that have sex with men in developing countries: Implications for HIV prevention. Crit Public Health. 1988;8.

13. Spradling PR, Richardson JT, Buchacz K, Moorman AC, Brooks JT. Prevalence of chronic hepatitis B virus infection among patients in the HIV Outpatient Study, 1996-2007. J Viral Hepat. 2010;17:87986.

14. Crowell TA, Gebo KA, Balagopal A, Fleishman JA, Agwu AL, Berry SA. Impact of hepatitis coinfection on hospitalization rates and causes in a multicenter cohort of persons living with HIV. J Acquir Immune Defic Syndr. 2014;65:429-37.

15. Crowell TA, Berry SA, Fleishman JA, LaRue RW, Korthuis PT, Nijhawan AE, et al. Impact of hepatitis coinfection on healthcare utilization among persons living with HIV. J Acquir Immune Defic Syndr. 2015;68:425-31.

16. Barth RE, Huijgen Q, Taljaard J, Hoepelman AIM. Hepatitis B/C and HIV in sub-Saharan Africa: An association between highly prevalent infectious diseases. A systematic review and meta-analysis. Int J Infect Dis. 2010;14:e1024-31.

17. Easterbrook P, Sands A, Harmanci H. Challenges and priorities in the management of HIV/HBV and HIV/HCV coinfection in resource-limited settings. Semin Liver Dis. 2012;32:147-57.

18. Musa BM, Bussell S, Borodo MM, Samaila AA, Femi OL. Prevalence of hepatitis B virus infection in Nigeria , 2000-2013: A systematic review and meta-analysis. Niger J Clin Pract. 2015;18.

19. Onyekwere CA, Hameed L. Hepatitis B and C virus prevalence and association with demographics: Report of population screening in Nigeria. Trop Doct. 2015;45:231-5.

20. Kooffreh-Ada M, Okpokam DC, Okaormhe ZA, Nna VU. Frequency of Hepatitis B and C Co-Infection in Chronic Liver Disease Patients in Calabar , Cross River State, Nigeria. Niger J Physiol Sci. 2016;31 June:43-7.

21. Omatola CA, Idofe J, Okolo MO, Adejo PO, Maina MM, Oyiguh JA. Seroprevalence of HBV among people living with HIV in Anyigba , Kogi State, Nigeria. Afr Health Sci. 2019;19:1938-46.

22. Tremeau-Bravard A, Ogbukagu IC, Ticao CJ, Abubakar JJ. Seroprevalence of hepatitis B and C infection among the HIV-positive population in Abuja, Nigeria. Afr Health Sci. 2012;12:312-7.

23. Omatola CA, Onoja BA, Thomas T. High Rate of Hepatitis B Virus Surface Antigenemia Among People Living with HIV/AIDS in Kakuri, Kaduna, North West Nigeria. Viral Immunol. 2017;30:516-21.

24. Adebajo SB, Myers T. Prevalence and correlates of HIV, syphilis, hepatitis $B$, hepatitis $C$ infections and sexual behaviours of men who have sex with men in two cities in Nigeria. Dalla Lana School of Public Health, University of Toronto; 2014. 
25. Charurat ME, Emmanuel B, Akolo C, Keshinro B, Nowak RG, Kennedy S, et al. Uptake of treatment as prevention for HIV and continuum of care among HIV-positive men who have sex with men in Nigeria. J Acquir Immune Defic Syndr. 2015;68 Suppl 2:S114-23.

26. Keshinro B, Crowell TA, Nowak RG, Adebajo S, Peel S, Gaydos CA, et al. High prevalence of HIV, chlamydia and gonorrhoea among men who have sex with men and transgender women attending trusted community centres in Abuja and Lagos, Nigeria. J Int AIDS Soc. 2016;19:21270. doi:10.7448/IAS.19.1.21270.

27. Federal Ministry of Health, National AIDS control program. Federal Republic of Nigeria National Guidelines for HIV Prevention Treatment and Care 2016). 2016.

28. Zhang Z. Model building strategy for logistic regression: purposeful selection. Ann Transl Med. 2009;4:4-10.

29. Soares CC, Georg I, Lampe E, Lewis L, Morgado MG, Nicol AF, et al. HIV-1, HBV, HCV, HTLV, HPV$16 / 18$, and Treponema pallidum infections in a sample of Brazilian men who have sex with men. PLoS One. 2014;9.

30. Mmbaga EJ, Moen K, Makyao N, Mpembeni R, Leshabari MT. HIV and STI s among men who have sex with men in Dodoma municipality , Tanzania: a cross-sectional study. 2017;:314-9.

31. Stockdale AJ, Mitambo C, Everett D, Geretti AM, Gordon MA. Epidemiology of hepatitis B, C and D in Malawi: systematic review. BMC Infect Dis. 2018;18:1-10.

32. Falade-Nwulia O, Seaberg EC, Snider AE, Rinaldo CR, Phair J, Witt MD, et al. Incident hepatitis B virus infection in HIV-infected and HIV-uninfected men who have sex with men from Pre-HAART to HAART periods: A cohort study. Ann Intern Med. 2015;163:673-80.

33. Shilaih M, Marzel A, Scherrer AU, Braun DL, Kovari H, Rougemont M, et al. Dually Active HIV / HBV Antiretrovirals as Protection Against Incident Hepatitis B Infections: Potential for Prophylaxis. J Infect Dis. 2016;214:599-606.

34. World Health Organization. Interim guidelines: Updated First- Line and Second Line Antiretroviral therapy and Post- exposure prophylaxis and recommendation on Early Infant Diagnosis of HIV. 2018.

35. Manne V, Gochanour E, Kowdley K V. Current perspectives into the evaluation and management of hepatitis B: a review. HepatoBiliary Surg Nutr. 2019;8:361-9.

36. Wong WWLL, Pechivanoglou P, Wong J, Bielecki JM, Haines A, Erman A, et al. Antiviral treatment for treatment-naïve chronic hepatitis B: Systematic review and network meta-analysis of randomized controlled trials. Syst Rev. 2019;8:1-16.

37. Chihota B V, Wandeler G, Chilengi R, Mulenga L, Chung RT, Bhattacharya D, et al. High Rates of Hepatitis B Virus ( HBV ) Functional Cure Among Human Immunodeficiency Virus-HBV Coinfected Patients on Antiretroviral Therapy in Zambia. J Infect Dis. 2020;221.

38. Nowak RG, Mitchell A, Crowell TA, Liu H, Ketende S, Ramadhani HO, et al. Individual and Sexual Network Predictors of HIV Incidence Among Men Who Have Sex With Men in Nigeria. J Acquir Immune Defic Syndr. 2019;80:444-53. 
39. Crowell TA, Baral SD, Schwartz S, Nowak RG, Kokogho A, Adebajo S, et al. Time to change the paradigm: limited condom and lubricant use among Nigerian men who have sex with men and transgender women despite availability and counseling. Ann Epidemiol. 2019;31:11-19.e3.

40. Emmanuel G, Folayan MO, Ochonye B, Umoh P, Wasiu B, Nkom M, et al. HIV sexual risk behavior and preferred HIV prevention service outlet by men who have sex with men in Nigeria. BMC Health Serv Res. 2019;:1-10.

41. Ophori EA, Tula MY, Azih A V, Okojie R, Ikpo EP. Current trends of immunization in Nigeria: Prospect and challenges. Trop Med Health. 2014;42:67-75.

42. Jansen $\mathrm{K}$, Thamm M, Bock CT, Scheufele R, Kücherer C, Muenstermann D, et al. High prevalence and high incidence of coinfection with Hepatitis $B$, Hepatitis $C$, and syphilis and low rate of effective vaccination against Hepatitis $B$ in HIVPositive men who have sex with men with known date of HIV seroconversion in Germany. PLoS One. 2015;10:1-18.

43. Nielsen US, Simonsen AB, Rensen $H \varnothing$, Larsen CS, Erikstrup C. Hepatitis B infection and vaccination coverage in men who have sex with men consulting a Danish venereal disease clinic. Infect Dis (Auckl). 2015; August 2014:517-22.

\section{Tables}


Table 1. Demographic, behavioral and clinical characteristics of Nigerian MSM and TGW with and without Hepatitis B virus

\begin{tabular}{|c|c|c|c|}
\hline & $\begin{array}{l}\text { HBV+ } \\
\mathrm{N}=69 \\
\mathrm{n}(\%)\end{array}$ & $\begin{array}{c}\text { HBV- } \\
\mathrm{N}=648 \\
\mathrm{n}(\%)\end{array}$ & $P^{*}$ \\
\hline HIV & & & 0.77 \\
\hline Uninfected & $28(10)$ & $251(90)$ & \\
\hline Infected & $41 \quad(9)$ & $397(91)$ & \\
\hline Age ( Years) & & & 0.51 \\
\hline $16-20$ & 12 (13) & 81 (87) & \\
\hline $20-25$ & $32 \quad(9)$ & $310(91)$ & \\
\hline $25-60$ & $25 \quad(9)$ & $257(91)$ & \\
\hline Site & & & 0.19 \\
\hline Abuja & 49 (11) & 408 ( 89) & \\
\hline Lagos & $20 \quad(8)$ & $240(92)$ & \\
\hline Education $\dagger$ & & & 0.09 \\
\hline Primary & $7(15)$ & 40 (85) & \\
\hline Secondary & 44 (11) & 373 (89) & \\
\hline Tertiary & $16(6)$ & $231(94)$ & \\
\hline Marital status $\dagger$ & & & 0.10 \\
\hline Never married & $56 \quad(9)$ & 567 (91) & \\
\hline Ever married & $9(12)$ & 65 (88) & \\
\hline Other & $4(24)$ & $13(76)$ & \\
\hline \multicolumn{4}{|c|}{ Willingness to access healthcare services $\dagger$} \\
\hline No & $24 \quad(9)$ & $238(91)$ & 0.74 \\
\hline Yes & $45(10)$ & $408(90)$ & \\
\hline HBV vaccination $\dagger$ & & & 0.14 \\
\hline No & $65(10)$ & $589(90)$ & \\
\hline Yes & $1 \quad(3)$ & 37 (97) & \\
\hline Sexual orientation $\dagger$ & & & 0.08 \\
\hline Homosexual & 31 (12) & $224(88)$ & \\
\hline Bisexual & $37 \quad(8)$ & $421(92)$ & \\
\hline Sexual position $\dagger$ & & & 0.83 \\
\hline Any receptive & $49 \quad(9)$ & 470 (91) & \\
\hline Only insertive & 17 (10) & $153(90)$ & \\
\hline Condom use during last sex† & & & $<0.01$ \\
\hline Yes & $33 \quad(7)$ & 426 (93) & \\
\hline No & 36 (14) & $216(86)$ & \\
\hline Concurrent sexual partners $\dagger$ & & & 0.98 \\
\hline No & 19 (10) & 177 (90) & \\
\hline Yes & 50 (10) & 469 (90) & \\
\hline \multicolumn{4}{|c|}{ Prevalent urethral and/or rectal Neisseria gonorrhoeae or Chlamydia trachomatis } \\
\hline No & $51(11)$ & 433 (89) & 0.23 \\
\hline Yes & $18 \quad(8)$ & 215 (92) & \\
\hline \multicolumn{4}{|c|}{ Vaginal sex partners in past yeart } \\
\hline No & 21 (13) & 141 (87) & 0.11 \\
\hline Yes & $48 \quad(9)$ & 500 (91) & \\
\hline
\end{tabular}

Abbreviations: MSM, men who have sex with men; TGW, transgender women; $p, p$-value;

HBV, hepatitis B virus.

† ' $\mathrm{n}$ ' may not add up to ' $\mathrm{N}$ ' because of missing values.

*Pearson's Chi-square and Fisher's exact tests. Bolded indicate $\mathrm{p}<0.05$. 
Page 15/17 
Table 2. Multivariable analysis of HIV and factors associated with HBV in Nigerian MSM \&TGW

Unadjusted OR

$(95 \% \mathrm{CI})$
Adjusted OR*

$(95 \% \mathrm{CI})$

\begin{tabular}{lll}
\hline HIV $\begin{array}{l}\text { Ref } \\
\text { uninfected } \\
\text { infected }\end{array}$ & $0.93(0.56-1.54)$ & $\begin{array}{c}\text { Ref } \\
0.95(0.65-1.59)\end{array}$ \\
\hline Age (Years) & $1.52(0.73-3.17)$ & \\
$16-20$ & $1.06(0.61-1.83) \quad$ Ref & \\
$20-25$ & & \\
$25-60$ & $1.44(0.84-2.48)$ & \\
\hline Site & Ref & \\
Abuja & Ref & \\
Lagos & $0.67(0.28-1.60)$ & \\
\hline Education & $0.40(0.15-1.02)$ & \\
Primary & & \\
Secondary & Ref & \\
Tertiary & $1.40(0.66-2.97)$ & \\
\hline Marital status & $3.18(0.98-9.88)$ & \\
Never Married & & \\
Ever Married & & \\
Other & & \\
\hline
\end{tabular}

Willingness to access health services

No Ref

Yes $\quad 0.94(0.54-1.54)$

HBV vaccination

No Ref

Yes $0.25(0.03-1.82)$

Sexual orientation

Homosexual $\quad 1.58(0.95-2.60)$

Bisexual Ref

$1.53(0.92-2.55)$

Ref

Sexual Position

Any receptive $\quad 0.94(0.52-1.68) \quad$ Ref

Only Insertive

Condom use at last anal sex

Yes

Ref

No $\quad 2.15(1.30-3.55)$

Ref

$2.18(1.31-3.60)$

Concurrent sexual partners

No

Ref

Yes $\quad 0.99(0.57-1.73)$

Prevalent urethral and/or rectal NG or CT

No Ref

Yes $\quad 0.71(0.40-1.24)$

Vaginal sex partners in past year

No

Ref

Yes

$0.65(0.37-1.11)$

Abbreviations: MSM, men who have sex with men; TGW, transgender women; Ref, reference; NG, Neisseria gonorrhoeae; CT, Chlamydia trachomatis.

Logistic regression was used to estimate odds ratios and 95\% confidence intervals. Bolded indicates significance at p-value $<0.05$.

*The final model was adjusted for condom use during last anal sex and sexual orientation. 


\section{Supplementary Files}

This is a list of supplementary files associated with this preprint. Click to download.

- AdeyemiSTROBEchecklistcrosssectional021820.doc 